\title{
Adicción al móvil en alumnos de secundaria: efectos en la convivencia
}

\author{
Noelia Flores Robaina ${ }^{1}$, Cristina Jenaro Río ${ }^{1}$, Francisca González Gi1 ${ }^{1}$, \\ Elena Martín Pastor ${ }^{1}$ y Raquel Poy Castro ${ }^{2}$ \\ ${ }^{1}$ Universidad de Salamanca, ${ }^{2}$ Universidad de León (España)
}

El objetivo general del presente estudio ha sido evaluar los patrones de uso del teléfono móvil en población adolescente escolarizada en centros de Educación Secundaria. De modo adicional, hemos evaluado correlatos psicológicos (ansiedad, depresión) y conductuales y asociación con rendimiento escolar. Para la realización del estudio se contó con 528 alumnos, con edades entre los 12 y los 19 años, pertenecientes a cinco centros educativos, cuatro públicos y uno concertado. Los instrumentos utilizados fueron el Cuestionario de Evaluación de la Depresión de Beck (BDI), el Cuestionario de Evaluación de la Ansiedad de Beck (BAI), las escalas de baja autoestima y problemas escolares del Cuestionario de Personalidad MMPI-A, y el Cuestionario para evaluar el uso excesivo del Móvil (COS). Los resultados indican que un porcentaje significativo de estudiantes que abusan del teléfono móvil muestran sintomatología depresiva y ansiógena y baja autoestima. Un $14.8 \%$ de los participantes obtiene puntuaciones que les sitúa en un rango de problemas escolares elevado o alto, a su vez vinculado con un uso patológico del teléfono móvil. Todos estos resultados ponen de manifiesto la necesidad de plantear intervenciones que reduzcan patrones conductuales desajustados y mejoren la convivencia escolar.

Palabras clave: Adicciones, convivencia, teléfono móvil.

Mobile Addiction in secondary school students: effects in coexistence. The objective of this study was to evaluate the patterns of mobile phone use among in secondary school students. Further, we assessed psychological (anxiety, depression) and behavioral correlates and association with school performance. To carry out the study we had 528 students, aged between 12 and 19 years, belonging to five schools, four public and one grant-aided private school. The instruments used were the Beck Depression Inventory (BDI), the Beck Anxiety Inventory (BAI), the low self-esteem and the school problems subscales of the MMPI-A Personality Questionnaire, and the Mobile Overuse Scale (COS). The results indicate that a significant percentage of students who abuse cellphones show anxious and depressive symptoms, and low self-esteem. Of participants, $14.8 \%$ obtained scores placing them in a range of medium-high to high for school problems associated to pathological use of the mobile phone. All these results highlight the need to establish interventions to reduce maladjusted behavioural patterns and improve school coexistence.

Key words: Addiction, coexistence, mobile phone.

Correspondencia: Noelia Flores Robaina. Facultad de Psicología. Universidad de Salamanca. Avda. de la Merced, 109-131. C.P. 37005. Salamanca (España).E-mail: nrobaina@usal.es 
Si bien la sociedad actual no podría entenderse sin el uso de las nuevas tecnologías de la información y de la comunicación (NTIC), y existe un acuerdo en reconocer sus ventajas, un creciente número de investigadores se centra en valorar los riesgos que entraña la tecnología de la información en la salud física y emocional, especialmente en jóvenes (Weare, 2004).

Cada vez son mayores las posibilidades de acceso a dichas herramientas y también cada vez es mayor su uso. Por ejemplo, respecto al uso de los teléfonos móviles y más concretamente de los mensajes de texto SMS, en los últimos cinco años se ha experimentado un claro incremento en su empleo y este medio de comunicación parece que continuará siendo el preferido frente a otras alternativas como son los mensajes multimedia, las llamadas telefónicas e incluso los contactos o interacciones cara a cara (Reid y Reid, 2004). De hecho, según Pedrero, Rodríguez y Ruiz (2012), el teléfono móvil ha pasado de ser un mero instrumento de comunicación interpersonal a convertirse en una plataforma de creciente complejidad que impacta en la vida de las personas en unas condiciones difícilmente comprensibles tan sólo unos años atrás. Por tanto, si bien en un principio cabía considerar al móvil como un teléfono inalámbrico privado, ahora es sólo una de sus múltiples utilidades, debiendo considerarse en la actualidad como una plataforma que oferta servicios variados, especialmente dirigidos a los más jóvenes, que no ha acabado de incorporar aún todas las funcionalidades posibles (Buchinger, Kriglstein, Brandt y Hlavacs, 2011).

El uso excesivo de estas herramientas ha hecho que algunos autores (p.e. Baruch, 2001) aludan a la "sociedad autista" para referirse a una sociedad caracterizada por la ausencia de intercambios sociales cara a cara y la puesta en marcha de conductas compulsivas relacionadas con dichas tecnologías. De acuerdo con Griffiths (2004), para muchas personas el concepto de adicción se relaciona con la ingesta de drogas. No obstante, cada vez más se reconoce la existencia de numerosas conductas potencialmente adictivas que no implican la ingesta de sustancias y que implican la puesta en marcha de una conducta con una frecuencia, intensidad o duración más elevada de lo considerado saludable.

Paulatinamente van apareciendo estudios que aluden a trastornos psicológicos y problemas conductuales relacionados con el uso abusivo de las NTIC. Así, respecto al uso del teléfono móvil, si bien los estudios relacionados con los problemas psicológicos asociados a su uso excesivo (tanto para realizar llamadas telefónicas, como para enviar mensajes de texto o acceder a juegos) son aún muy escasos, se ha encontrado una relación entre esta conducta y otras que suponen un riesgo para la salud, como son el consumo de alcohol y/o tabaco (Leena, Romi y Arja, 2005). Otros trabajos realizados con estudiantes universitarios constatan una estrecha relación entre el excesivo envío de SMS (servicio de mensajes breves) y el estrés (Jenaro et al., 2007; Thomeée, Eklöf, Gustafsson, Nilsson y Hagberg, 2007). Por su parte, en un estudio realizado con 
población adolescente también se puso de manifiesto que el consultar frecuentemente la pantalla del teléfono móvil para ver si se había recibido alguna llamada o SMS era la conducta que mayor dependencia y malestar generaba en esta población (Labrador y Villadongos, 2010).

Uno de los peligros a los que se suele aludir frecuentemente es a las dificultades en el adecuado desarrollo de habilidades sociales, que caracteriza a estos jóvenes (Weare, 2004). También se ha encontrado una relación entre el uso de NTIC por parte de estudiantes universitarios y niveles de estrés percibido, síntomas de depresión y trastornos del sueño (p.e. Thomeée, Eklöf, Gustafsson, Nilsson y Hagberg, 2007). Además, se han constatado los efectos negativos de los videojuegos de contenidos violentos en las conductas hostiles y agresivas de los adolescentes (Gentile, Lynch, Linder y Walsj, 2004).

Los trabajos en este campo están además proporcionando interesantes evidencias empíricas que, a su vez, permiten formular o reformular planteamientos teóricos en este campo. Así, se alude a una serie de factores comunes a la adquisición y mantenimiento de las diferentes conductas adictivas (Echeburúa, 1994). Dichos factores se relacionan no sólo con variables individuales sino también con factores ambientales, como pueden ser la aceptación social y disponibilidad ambiental de estos estímulos.

A la vista de lo anteriormente expuesto, con el presente trabajo pretendemos contribuir a incrementar los conocimientos referentes a estas nuevas adicciones, concretamente la del teléfono móvil en estudiantes de secundaria. Para ello nos hemos marcado los siguientes objetivos: 1) Evaluar la presencia de conductas adictivas relacionadas con el uso del teléfono móvil en estudiantes de Secundaria; 2) Identificar correlatos psicológicos y conductuales asociados al uso abusivo del teléfono móvil; y 3) Evaluar el impacto del uso abusivo del móvil en variables escolares.

\section{MÉTODO}

\section{Procedimiento}

Los datos fueron recogidos durante el primer semestre de 2011. El proceso para ello se inició contactando a través del correo electrónico con todos los centros de Educación Secundaria públicos y algunos concertados y privados de Salamanca capital. Transcurridos unos días de esta notificación se realizaron varios contactos telefónicos con cada centro para concretar su posible participación y los detalles de la misma.

Una vez lograda la participación, se establecieron los horarios para la aplicación de cuestionarios. Dicha aplicación se realizó conjuntamente a todos los alumnos de cada aula de secundaria. Todos los participantes colaboraron de manera voluntaria en el estudio. A todos ellos se les garantizó, además, el anonimato y la confidencialidad en las respuestas emitidas. Los miembros del equipo investigador 
asumieron el compromiso de enviar un informe general a los centros con los resultados obtenidos en el estudio.

\section{Participantes}

Los participantes, un total de 528, se distribuyen en 280 mujeres (53\%) y 246 hombres $(46.6 \%)$, existiendo dos personas $(0.4 \%)$ que no incluyen esta información. Las edades de los estudiantes oscilan entre los 12 y los 19 años, con un ligero predominio de quienes tienen 14 años (19.7\%), seguido de quienes tienen 15 o 16 años (18.2 y 15.5\%).

En cuanto al tipo de centro en el que se encuentran escolarizados, cabe destacar un predominio de los centros públicos $(80.9 \%)$, frente a los privados $(10.7 \%)$ y concertados $(8.4 \%)$.

Respecto al curso en el que estudian los participantes, conviene destacar que 93 estudiantes se caracterizan por encontrarse en $1^{\circ}$ de la ESO (17.6\%); 89 en $2^{\circ}(16.9 \%)$ 134 en $3^{\circ}(25.4 \%)$ y 68 en $4^{\circ}(12.9)$ frente a 92 alumnos que pertenecen a $1^{\circ}$ de Bachillerato (17.4\%) y 52 que se encuentran cursando $2^{\circ}$ curso de Bachillerato $(9.8 \%)$, respectivamente.

\section{Instrumentos}

En primer lugar, y para evaluar la adicción al teléfono móvil se utilizó la Escala de Uso Excesivo de teléfono móvil (COS) diseñada por Jenaro, Flores, GómezVela, González-Gil y Caballo (2007). Se trata de un instrumento compuesto por 23 ítems que evalúa conductas, pensamientos y sentimientos relacionados con el uso y abuso del teléfono móvil. Los participantes deben indicar en una escala tipo Likert de 6 puntos, que oscila de 1 (nunca) a 6 (siempre), la frecuencia en que experimentan el contenido de cada uno de los enunciados. Un ejemplo de ítem sería: “ ¿Se siente preocupado por si ha recibido alguna llamada o mensaje y piensa en ello cuando tiene el móvil apagado?”. Dicho instrumento está basado en los criterios diagnósticos propuestos por el DSM-IV (4th ed.; DSM-IV; American Psychiatric Association, 1994) para el juego patológico, si bien se trata de evaluar un uso excesivo más que un trastorno adictivo. La fiabilidad de dicho instrumento fue de alfa $=0.87$.

En segundo lugar, para evaluar los correlatos psicológicos se utilizaron las versiones españolas del Cuestionario de Evaluación de la Depresión BDI y el Cuestionario de Evaluación de la Ansiedad BAI de Beck (Beck, Rush, Shaw y Emery, 1979; Beck, Brown, Epstein, y Steer, 1988). Respecto al BDI, consta de 21 ítems con cuatro respuestas (de 0 a 3 ) para cada síntoma y una puntuación total que abarca de 0 a 63. Las categorías de severidad se clasifican en: normal (de 0 a 9 puntos), ligera (de 10 a 15), moderada (de 16 a 23) y severa (de 24-63). De las diversas versiones españolas, hemos empleado la adaptación de Sanz y Vázquez (1998) (Sanz y Navarro, 2003). El punto de corte empleado en esta versión y recomendado por el autor original es de 18. 
Este es, por tanto, el punto de corte que hemos empleado en este trabajo para distinguir entre población clínica o no, en cuanto a depresión. La fiabilidad del instrumento fue contrastada mediante el alfa de Cronbach y los resultados (alfa=0.88) apoyaron su fiabilidad. Por su parte el BAI también consta de 21 ítems, que evalúan la intensidad de la ansiedad clínica de modo independiente de la depresión. Las puntuaciones oscilan de 0 a 62. De acuerdo con algunos estudios (e.g. Somoza, Steer, Beck, y Clarke, 1994), el punto de corte de las muestras clínicas es 14. Éste es el punto empleado en el presente estudio. Hemos calculado también el alfa de Cronbach y los resultados (alfa=0.87), apoyan su fiabilidad.

En tercer lugar, hemos empleado el MMPI-A (Butcher, Willians, Graham, Archer, Tellegen, Ben-Porathe, y Kaemmer, 1992). Este instrumento Permite evaluar distintos rasgos de la personalidad normal y patológica en adolescentes. La adaptación española (Jiménez-Gómez y Ávila-Espada, 2003) presenta adecuadas propiedades psicométricas. Concretamente, se han aplicado las escalas de baja autoestima y problemas escolares de este instrumento. La primera está compuesta por 18 ítems. Puntuaciones elevadas denotan opiniones negativas sobre sí mismos. Las personas pueden considerarse muy influenciables por los demás e incapaces de resolver problemas interpersonales. La segunda escala, está compuesta por 20 ítems cuyas puntuaciones altas revelan distintos tipos de dificultades como experiencias de fracaso en los aprendizajes así como dificultades de ajuste al medio escolar.

\section{RESULTADOS}

Presentamos en primer lugar los resultados relativos a nuestro primer objetivo, esto es, evaluar la posible presencia de conductas adictivas, relacionadas con el uso del teléfono móvil, en estudiantes de educación secundaria.

Así, en la tabla 1 se detallan los patrones de utilización de teléfono móvil. En este sentido, se puede apreciar cómo una amplia mayoría (96\%) indican tener teléfono móvil y cómo incluso un $19.3 \%$ indican tener más de uno. Por lo general $(54.2 \%)$ se trata de teléfonos con tarjeta de recarga y se emplean para las actividades ordinarias: llamar por teléfono o enviar SMS (95.1 y 90.9\% respectivamente). No obstante, un alto porcentaje de los encuestados lo utiliza como bluetooth $(73.1 \%)$ o para envío de MMS $(30.7 \%)$. Por lo general los estudiantes emplean el móvil menos de una hora al día $(51.5 \%)$ o entre 1 y 3 horas $(25.8 \%)$, y realizan un gasto mensual entre 10 y 40 euros.

Una vez presentados los patrones de uso, pasamos a analizar con más detalle los patrones de abuso o uso excesivo del teléfono móvil. Para ellos empleamos la Escala de Uso Excesivo del Teléfono Móvil (COS).

Como indicamos previamente, el instrumento se utiliza para identificar, en primer lugar, a quienes hacen un uso elevado vs. bajo (es decir, centiles 75 y $25 ; N=138$ 
y $N=130$, respectivamente), de este sistema, tomando como referencia la muestra estudiada. Por otro lado, utilizamos como punto de corte para diferenciar entre población clínica o no (es decir, usuarios patológicos o no) el cumplimiento de los criterios del DSM. Así, de acuerdo con los criterios DSM-IV, un 19.3\% $(N=102)$ de la muestra total se pueden considerar usuarios patológicos de Móvil.

Tabla 1. Patrones de uso del teléfono móvil

\begin{tabular}{|c|c|c|}
\hline & $N$ & $\%$ \\
\hline \multicolumn{3}{|l|}{ Teléfono móvil } \\
\hline Sí, más de uno & 102 & 19.3 \\
\hline Sí, uno & 388 & 73.5 \\
\hline Sí, compartido & 12 & 2.3 \\
\hline No & 21 & 4.0 \\
\hline N.R. & 5 & 0.9 \\
\hline \multicolumn{3}{|l|}{ Tipo de móvil } \\
\hline Con tarjeta de recarga & 286 & 54.2 \\
\hline Con contrato & 181 & 34.3 \\
\hline Ambos & 37 & 7.0 \\
\hline N.R. & 24 & 4.5 \\
\hline \multicolumn{3}{|l|}{ Usos del móvil } \\
\hline Realizar llamadas & 502 & 95.1 \\
\hline Envío de sms & 480 & 90.9 \\
\hline Descargas de tonos & 34 & 6.4 \\
\hline Descargas de juegos & 39 & 7.4 \\
\hline Descargas de fondos & 21 & 4.0 \\
\hline Para chatear (WAP) & 9 & 1.7 \\
\hline Videollamadas & 51 & 9.7 \\
\hline Como bluetooth & 386 & 73.1 \\
\hline Envío de mms & 162 & 30.7 \\
\hline \multicolumn{3}{|l|}{ Promedio diario de uso } \\
\hline Menos 1 hora & 272 & 51.5 \\
\hline 1-3 horas & 136 & 25.8 \\
\hline 4-5 horas & 32 & 6.1 \\
\hline Más de 5 horas & 65 & 12.3 \\
\hline N.R. & 23 & 4.4 \\
\hline \multicolumn{3}{|l|}{ Gasto mensual } \\
\hline Menos de 10 euros & 238 & 45.1 \\
\hline 10-20 euros & 167 & 31.6 \\
\hline $21-40$ euros & 59 & 11.2 \\
\hline 41-60 euros & 24 & 4.5 \\
\hline $61-80$ euros & 5 & 0.9 \\
\hline Más 80 euros & 13 & 2.5 \\
\hline NR & 22 & 4.2 \\
\hline
\end{tabular}

En la tabla 2 se observa además la existencia de participantes que cumplen diferente número de síntomas clínicos asociados al uso patológico del móvil, existiendo un predominio de quienes cumplen con un criterio (27.3\%).

Para considerar que un sujeto cumple con los criterios diagnósticos de uso patológico es necesario el cumplimiento de cinco o más síntomas. En nuestro caso, se observa cómo un $19.3 \%$ de los encuestados cumplen con los criterios diagnósticos. 
Tabla 2. Presencia de síntomas clínicos de uso de Móvil en la muestra analizada

\begin{tabular}{lcc}
\hline & $N$ & $\%$ \\
\hline Número de síntomas clínicos de Uso de Móvil & & \\
\hline Cero & 144 & 27.3 \\
\hline Uno & 108 & 20.5 \\
\hline Dos & 80 & 15.2 \\
\hline Tres & 47 & 8.9 \\
\hline Cuatro & 47 & 8.9 \\
\hline Cinco & 34 & 6.4 \\
\hline Seis & 36 & 6.8 \\
\hline Siete & 32 & 6.1 \\
\hline Síntomas de adicción al móvil & & \\
\hline Población no clínica & 426 & 80.7 \\
\hline Población clínica & 102 & 19.3 \\
\hline
\end{tabular}

Respecto a los bajos $v s$. altos usuarios de teléfonos móviles ( $N=138$ y $N=130$, respectivamente), el 25\% $(N=45)$ de los usuarios no patológicos de teléfonos móviles fueron clasificados como elevados usuarios, mientras que el 96.6\% (N=85) de los usuarios patológicos fueron clasificados correctamente como altos usuarios. En suma, es posible afirmar que la muestra objeto de estudio incluye más elevados usuarios que usuarios patológicos (Tabla 3).

Tabla 3. Comparación entre grupos altos y bajos, y grupos clínicos y no clínicos (Utilización de Móvil)

\begin{tabular}{lrrr}
\hline & Grupo Bajo & Grupo Alto & \multicolumn{1}{c}{ Total } \\
\hline Grupo No Clínico & $135(75 \%)$ & $45(25 \%)$ & $180(100 \%)$ \\
\hline Grupo Clínico & $3(3.4 \%)$ & $85(96.6 \%)$ & $88(100 \%)$ \\
\hline Total & $138(51.5 \%)$ & $130(48.5 \%)$ & $268(100 \%)$ \\
\hline
\end{tabular}

El segundo objetivo se centraba en identificar correlatos psicológicos y conductuales asociados al uso abusivo del teléfono móvil. Respecto a los correlatos psicológicos, es posible identificar cómo 166 (31.4\%) estudiantes de secundaria de nuestra muestra cumplen con los criterios diagnósticos de ansiedad según el BAI frente a $362(68.6 \%)$ de los alumnos que pueden considerarse población no clínica. Así mismo, respecto a la sintomatología depresiva evaluada a través del BDI, encontramos que un $25.8 \%$ de los participantes $(N=136)$ obtiene puntuaciones que le sitúan con un grado de depresión de leve a severo, frente al 74.2\% ( $N=392)$ que no presentan sintomatología depresiva clínicamente significativa. En cuanto a la autoestima, un $16 \%(N=84)$ presenta puntuaciones correspondientes a baja autoestima. En este sentido, los análisis chi cuadrado realizados para contrastar la presencia o ausencia de una asociación entre síntomas clínicos de ansiedad, depresión y baja autoestima y la ausencia o presencia de uso patológico del teléfono móvil, indicaron la presencia de una asociación significativa con dichas variables (Tabla 4).

Tabla 4. Significación de la asociación entre síntomas y uso patológico del móvil

\begin{tabular}{lcc}
\hline & $\chi^{2}$ & $p$ \\
\hline Ansiedad (BAI) & 16.162 & 0.000 \\
\hline Depresión (BDI) & 10.322 & 0.001 \\
\hline Baja Autoestima (LSE) & 14.918 & 0.001 \\
\hline
\end{tabular}


Respecto a los correlatos conductuales, en la tabla 5 se puede observar la presencia de conductas adictivas y saludables en la muestra de estudiantes de secundaria.

Por su parte, los análisis chi cuadrado realizados para contrastar la presencia o ausencia de uso patológico del móvil y las conductas adictivas, indicaron una asociación significativa con las variables fumar más de 20 cigarrillos $\left(\chi^{2}=10.299 ; p=0.004\right)$, beber alcohol de manera abusiva $\left(\chi^{2}=11.437 ; p=0.001\right)$ y jugar a máquinas tragaperras $\left(\chi^{2}=12.822 ; p=0.001\right)$. Así mismo, también se encontró asociación significativa con las conductas saludables de dormir horas suficientes $\left(\chi^{2}=6.254 ; p=0.009\right)$, realizar actividad física moderada $\left(\chi^{2}=4.019 ; p=0.030\right)$, practicar deporte o ejercicio al menos tres veces a la semana $\left(\chi^{2}=4.395 ; p=0.025\right)$ y destinar tiempo para el ocio $\left(\chi^{2}=4.964 ; p=0.021\right)$.

Tabla 5. Presencia de conductas adictivas y saludables en la muestra objeto de estudio

\begin{tabular}{lcc}
\hline & $N$ & $\%$ del total $(N=528)$ \\
\hline Conductas Adictivas & 22 & \\
\hline Fumar más de 20 cigarrillos al día & 49 & 4.2 \\
\hline Consumir sustancias & 75 & 9.3 \\
\hline Beber alcohol de manera abusiva & 20 & 14.2 \\
\hline Jugar a máquinas tragaperras o bingo, más de una vez a la semana & 75 & 14.2 \\
\hline Jugar a apuestas (lotería, bonoloto, quinielas, etc.) más de una vez a la semana & & \\
\hline Conductas Saludables & 358 & 67.8 \\
\hline Dormir horas suficientes & 333 & 63.1 \\
\hline Realizar actividad física moderada (p.e. caminar rápido) & 330 & 62.5 \\
\hline Practicar deportes o ejercicio al menos tres veces a la semana & 423 & 80.1 \\
\hline Destinar tiempo para el ocio & & \\
\hline
\end{tabular}

Finalmente, y en relación con nuestro tercer objetivo, esto es, evaluar el impacto del uso abusivo del móvil en variables escolares, en la tabla 5 exponemos la frecuencia de aparición de problemas escolares o no, según el MMPI-A. Se puede observar que un $14.8 \%$ de los participantes obtiene puntuaciones que les sitúa en un rango de problemas escolares elevado o alto. Por su parte, el análisis de la asociación entre la presencia o ausencia de problemas escolares y la utilización patológica del teléfono móvil puso de manifiesto la existencia de asociación entre ambas variables $\left(\chi^{2}=21.641 ; p=0.000\right)$.

Tabla 6. Problemas escolares

\begin{tabular}{|c|c|c|}
\hline & $N$ & $\%$ \\
\hline \multicolumn{3}{|l|}{ Problemas escolares } \\
\hline No Clínico & 295 & 55.9 \\
\hline Moderadamente elevado & 51 & 9.7 \\
\hline Alto & 27 & 5.1 \\
\hline
\end{tabular}

\section{DISCUSIÓN Y CONCLUSIONES}

Los resultados obtenidos en este estudio nos han permitido evidenciar cómo un $19.3 \%$ de los estudiantes de secundaria evaluados cumplen con los criterios de uso 
patológico del teléfono móvil. Conviene decir que este resultado coincide con las tasas de prevalencia encontradas en otros estudios realizados en nuestro país (Jenaro et al., 2007; Labrador y Villadangos, 2010; Sánchez-Martínez y Otero, 2009).

Por lo tanto, y pese a que este estudio no haya empleado una muestra seleccionada aleatoriamente, ni se haya empleado un sistema de muestreo que asegure la representatividad de los datos obtenidos, las elevadas tasas de uso patológico del teléfono móvil son similares a las encontradas en otros estudios (Carbonell, Fúster, Chamarro y Oberst, 2012) y merecen ser tenidas en cuenta, dada la dependencia emocional, falta de control e impacto adicional negativo en ámbitos sociales, laborales o educativos, que estas conductas pueden ocasionar.

También hemos constatado cómo un $31.4 \%$ de los estudiantes cumple con los criterios de ansiedad clínica; un $25.8 \%$ de los participantes obtiene puntuaciones que le sitúan con un grado de depresión de leve a severo y un $16 \%$ de los estudiantes presentan baja autoestima. Por su parte, el análisis de la posible asociación entre el uso excesivo o no del teléfono móvil y las mencionadas alteraciones indicaron la existencia de una asociación significativa con la ansiedad (medida según el BAI), la depresión (medida según el BDI) y la baja autoestima (según el MMPI-A). De tal forma que existe una mayor proporción de alumnos con elevada ansiedad, con elevada depresión y con baja autoestima, que al mismo tiempo hacen un uso excesivo del teléfono móvil. Estos datos apoyan lo obtenido en otras investigaciones (Billeux, Van der Linden y Rochat, 2008; Kim et al., 2006; Morahan-Martin y Schumacher, 2000; Whang, Lee y Chang, 2003; Young y Rogers, 1998).

En lo que se refiere a nuestro tercer objetivo, un $14.8 \%$ de los participantes obtiene puntuaciones que les sitúa en un rango de problemas escolares elevado o alto, a su vez vinculado con un uso patológico del teléfono móvil. Esto pone de manifiesto la necesidad de plantear intervenciones a nivel educativo que ayuden a solucionar este tipo de problemas con objeto de mejorar los problemas escolares que presentan estos estudiantes y dotarles de estrategias que puedan favorecer sus relaciones interpersonales y la convivencia en el ámbito escolar. Es necesario desarrollar, por tanto, estudios de detección precoz de aquellos alumnos que se puedan encontrar en riesgo de presentar un uso patológico, así como de realizar intervenciones psicológicas y educativas destinadas a enseñar comportamientos de uso de la NTIC saludables y que incidan especialmente en reducir las consecuencias psicológicas y escolares que estos problemas pueden generar en el adolescente.

No queremos terminar sin antes reflejar algunas limitaciones del presente estudio. En primer lugar, y como ya ha sido avanzado previamente, el procedimiento de selección muestral no nos permite generalizar los resultados obtenidos, ni hablar en sentido estricto de prevalencia de estos nuevos trastornos. En segundo lugar, es necesario llevar a cabo más estudios con muestras más amplias de usuarios patológicos de teléfono 
móvil, que nos permitan confirmar o refutar los presentes hallazgos. En tercer lugar, es necesario poner en marcha estudios longitudinales y estudios experimentales que nos ayuden a dar respuestas a algunas cuestiones aún sin resolver y que, en definitiva, contribuyan a reducir estos trastornos conductuales.

\section{REFERENCIAS}

American Psychiatric Association (1994). Diagnostic and statistical manual of mental disorders (4 $4^{\mathrm{a}}$ Ed.) (DSM-IV). Washington, D.C.: American Psychiatric Association.

Beck, A.T., Brown, G., Epstein, N. y Steer, R.A. (1988). An inventory for measuring clinical anxiety: Psychometric properties. Journal of Consulting and Clinical Psychology, 56(6), 893-897.

Beck, A.T., Rush, A.J., Shaw, B.F. y Emery, G. (1979). Cognitive therapy of depression. New York: Guilford Press. Beck., A.T. y Steer, R.A. (1990). Manual for the Beck Anxiety Inventory. San Antonio TX: Psychological Corporation.

Baruch, Y. (2001). The autistic society. Information y Management, 38, 129-136.

Billieux, J., Van der Linden, M. y Rochat, L. (2008). The role of impulsivity in actual and problematic use of the mobile phone. Applied Cognitive Psychology, 22, 1195-1210.

Butcher, J.N., Willians, C.L., Graham, J.R., Archer, R.P., Tellegen, A., Ben-Porathe, Y.S. y Kaemmer, B. (1992). Minnesota Multiphasic Personaltiy Inventory Adolescent (MMPIA): Manual for administration, scoring and interpretation. Minneapolis: University of Minnesota Press. (Traducción, adaptación al español por Jiménez-Gómez, F. y ÁvilaEspada, A. (2003). Madrid: TEA Ediciones).

Buchinger, S., Kriglstein, S., Brandt, S. y Hlavacs, H. (2011). A survey on user studies and technical aspects of mobile multimedia applications. Entertaiment Computing, 2, 175190.

Carbonell, X., Fúster, H., Chamarro, A. y Oberst, U. (2012). Adicción a internet y al móvil: una revisión de estudios empíricos españoles. Papeles del Psicólogo, 33, 82-89.

Echeburúa, E. (1994), Evaluación y tratamiento de los trastornos adictivos. Madrid. Terapia de Conducta y Salud.

Gentile, D.A., Lynch, P.J., Linder, J.R. y Walsj, D.A. (2004). The effects of violent video game habits on adolescent hostility, aggressive behaviors, and school performance. Journal of Adolescent, 27, 5-22.

Griffiths, M. (2004). Behavioural addiction: an issue for everybody? Employee Counselling Today: The Journal of Workplace Learning, 8(3), 19-25.

Jenaro, C., Flores, N., Gómez-Vela, M., González-Gil, F. y Caballo, C. (2007). Problematic internet and cell-phone use: Psychological, behavioral, and health correlates. Addiction Research \& Theory, 15, 309-320.

Kim, K., Ryu, E., Chon, M.Y., Yeun, E.J., Choi, S.J, Seo, J.S. y Nam, B.W. (2006). Internet addiction in Korean adolescents and its relation to depression and suicidal ideation: A questionnaire survey. International Journal of Nursing Studies, 43, 185-192.

Labrador, F.J. y Villadongos, S. (2010). Menores y Nuevas Tecnologías: conductas indicadoras de un posible problema de adicción. Psicothema, 22, 180-188.

Leena, K., Tomi, L. y Arja, R. (2005). Intensity of mobile phone use and health compromising behaviours how is information and communication technology connected to healthrelated lifestyle in adolescence? Journal of Adolescent, 28, 35-47. 
Morahan-Martin, J y Schumacher, P. (2000). Incidence and correlates of pathological Internet use among college students. Computers in Human Behavior, 16, 13-29.

Pedrero, E., Rodríguez, M.T. y Ruíz, J.M. (2012). Adicción o abuso del teléfono móvil. Revisión de la literatura. Adicciones, 24, 139-152.

Reid, F.J.M. y Reid, D.J. (2004). Text appeal: the psychology of SMS texting and its implications for the design of mobile phone interfaces. Campus-Wide Information Systems, 21(5), 196-200.

Sánchez-Martínez, M. y Otero, A. (2009). Factors associated with cell phone use in adolescents in the Community of Madrid (Spain). Cyberpsychology and Behavior, 12, 131-137.

Sanz, J. y Navarro, M.E. (2003). Propiedades psicométricas de una versión española del Inventario de Ansiedad de Beck (BAI) en estudiantes universitarios. Ansiedad y Estrés, 9, 59-84.

Sanz, J. y Vázquez, C. (1998). Fiabilidad, validez y datos normativos del Inventario para la depresión de Beck. Psicothema, 10, 303-318.

Somoza, E., Steer, R.A., Beck, A.T. y Clarke, D.A. (1994). Differentiating major depression and panic disorders by self-report and clinical rating scales: ROC analysis and information theory. Behaviour Research and Theory, 32, 771- 782.

Thomeée, S., Eklöf, M., Gustafsson, E., Nilsson, R. y Hagberg, M. (2007). Prevalence of perceived stress, symptoms of depression and sleep disturbances in relation to information and communication technology (ICT) use among young adults -an explorative prospective study. Computers in Human Behavior, 23, 1300-1321.

Whang, L.S.M., Lee, S. y Chang, G. (2003). Internet over users' psychological profiles: A behavior sampling analysis on Internet addiction. CyberPsychology and Behavior, 6, 143-150.

Weare, K. (2004). What impact is having information techonolgy on our young people's health and well-being. Health Education, 104, 129-131.

Young, K.S. y Rogers, R.C. (1998). The relationship between depression and Internet addiction. CyberPsychology and Behavior, 1, 25-28.

Recibido: 25 de febrero de 2013

Recepción Modificaciones: 8 de marzo de 2013

Aceptado: 19 de agosto de 2013 